\title{
Improvements towards the inclusion of magnetic effects in large-scale multislice calculations of elastic electron scattering
}

\author{
Keenan Lyon and Jan Rusz \\ Uppsala University, Uppsala, Sweden
}

\section{Introduction}

The engineering, design, and exploration of novel magnetic materials necessitates characterization methods capable of rendering the behaviour of these materials at the atomic scale. Recent progress in the development of electron beam monochromators has made it possible for the ever-versatile transmission electron microscope (TEM) to probe low energy excitations at this scale. Within the TEM setup, electron holography, Lorentz microscopy, differential phase contrast microscopy, and electron magnetic circular dichroism have all been put forward as approaches to study magnetism in materials. At the theoretical heart of many of these TEM approaches lies the multislice method [1], which simulates the propagation of the incident electron wavefunction through a crystal. While this method is wellestablished in the literature, the inclusion of magnetic effects poses novel challenges to both the efficiency and implementation of the multislice method for larger systems. This abstract presents our recent efforts to face these challenges.

\section{Objective}

First, as inclusion of magnetic fields into the multislice method necessarily means working with the paraxial Pauli equation where the non-periodic magnetic vector potential plays a role [2], the 2D Fourier transform utilized in conventional multislice calculations to speed things up considerably can no longer be applied as before [1]. Our objective on this front is to introduce a formulation for the real space magnetic multislice that takes advantage of this speedup by including non-periodic magnetic contributions in a perturbative fashion. Second, as magnetic multislice calculations over large crystals require knowledge of the full magnetic fields with an accuracy near the level afforded by DFT calculations [2], a method is required analogous to electron form factors that can yield these fields without prohibitively heavy computation. The secondary objective is to therefore formulate a parametrization of magnetic fields for a variety of magnetic elements, allowing for the quick calculation of magnetic fields at any point in a crystal system.

3. Materials \& methods

We make use of the Pauli multislice framework presented in [2]. For the calculation of magnetic fields, DFT calculations are done in GPAW, from which the spin density can be used via the Poisson equation to obtain the magnetic field and vector potential [2].

DFT calculations are done for individual atoms with magnetic moments, and the magnetic fields and vector potentials are fit to quasi-dipole like expressions that satisfy symmetry relations and physical constraints on the summation of the field components over all space while avoiding the divergent behaviour near atom centres expected from a simplistic dipole approximation.

4. Results

While making use of the real space multislice methods requires an iterative procedure until a certain convergence criterion is reached that often requires up to ten iterations, our perturbative Pauli multislice implementation can be run without the use of any such iterative procedure, much like the conventional multislice. 
The parameterization of magnetic fields via our quasi-dipole approach can be accomplished with as few as nine parameters across many magnetic atoms yielding fits with extremely low errors. These parameterized magnetic fields from atomic systems can then be summed together for multiatomic systems. One such example of Ax on an $84 \AA \times 84 \AA$ slice of bcc Fe model generated with the atomistic spin dynamics code UppASD [3] constructed via the parameterized values is attached with this abstract.

5. Conclusion

In conclusion, the development of a perturbative approach to magnetic multislice simulations along with parameterization of magnetic fields and vector potentials in a way analogous to parametrization of atomic form factors allows for far more efficient calculation of elastic electron scattering in systems where magnetic effects are expected to play an important role.

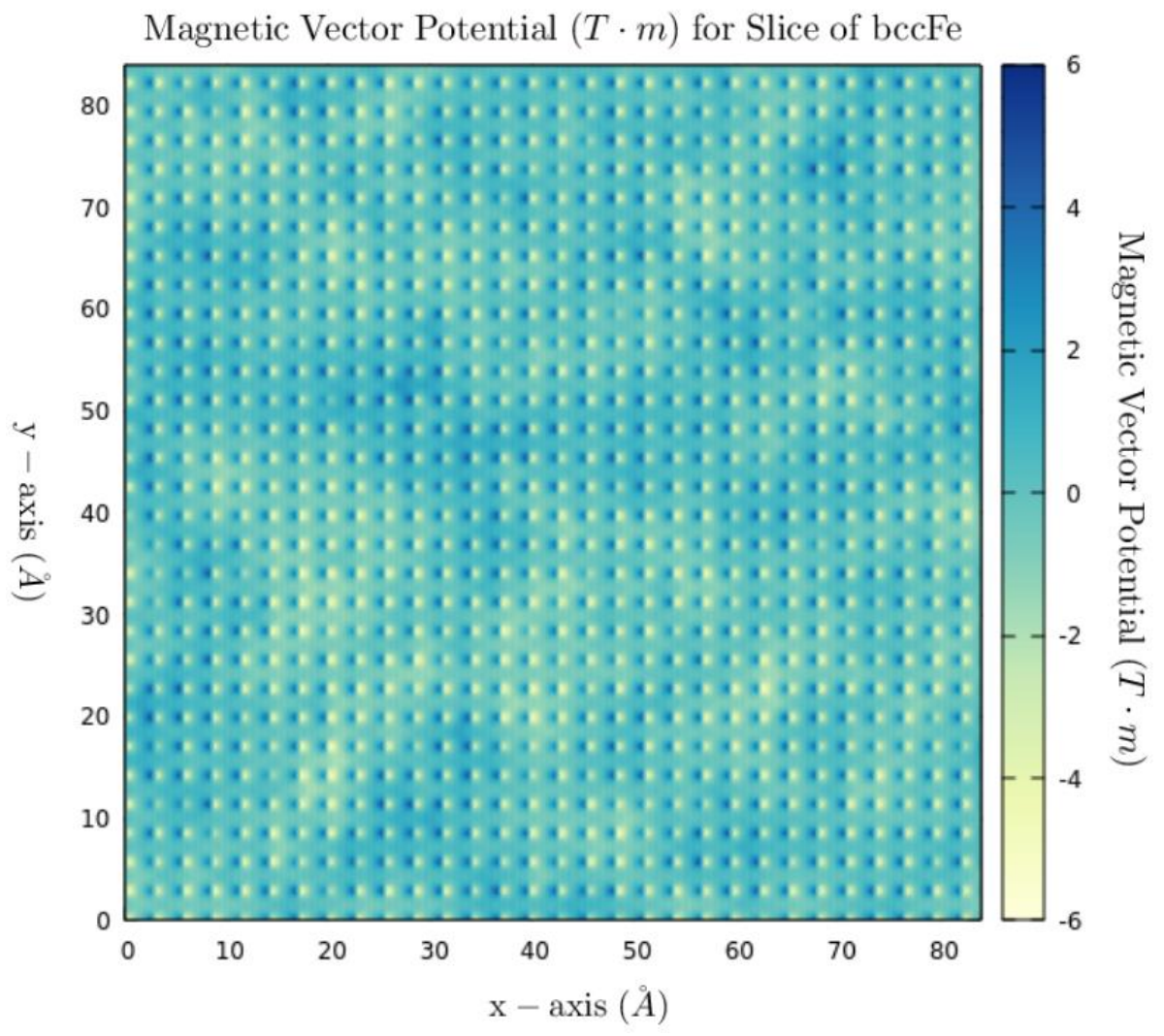

Figure 1. Heatmap in the $x y$-plane at $z=0$ for $A \_x$ for a supercell of bcc Fe of size $86 \AA \times 86 \AA \times 287 \AA$, with the magnetic vector potential generated from the parameterization of magnetic fields around the iron atom.

\section{References}

[1] E. J. Kirkland, Advanced Computing in Electron Microscopy (2nd edition), Springer, 2010.

[2] A. Edström, A. Lubk, J. Rusz, Phys. Rev. B94, 174414, 2016.

[3] O. Eriksson, A. Bergman, L. Bergqvist, and J. Hellsvik, Atomistic Spin Dynamics - Foundations and Applications, Oxford University Press, Oxford, 1st edition, 2017 